\title{
EDGE OF THE WEDGE THEOREM FOR TEMPERED ULTRAHYPERFUNCTIONS
}

\author{
E. BRÜNING AND S. NAGAMACHI
}

\begin{abstract}
Tempered ultrahyperfunctions do not have the same type of localization properties as Schwartz distributions or Sato hyperfunctions; but the localization properties seem to play an important role in the proofs of the various versions of the edge of the wedge theorem. Thus, for tempered ultrahyperfunctions, one finds a global form of this result in the literature, but no local version.

In this paper we propose and prove a formulation of the edge of the wedge theorem for tempered ultrahyperfunctions, both in global and local form. We explain our strategy first for the one variable case. We argue that in view of the cohomological definition of hyperfunctions and ultrahyperfunctions, the global form of the edge of the wedge theorem is not surprising at all.
\end{abstract}

\section{Contents}

1. Introduction

2. Preliminaries

2.1. Global and local versions of the EOW Theorem

2.2. The one dimensional case

3. Global edge of the wedge theorem

4. Local edge of the wedge theorem

References

\section{INTRODUCTION}

Nowadays, i.e., about fifty years after its discovery, there are many versions of the 'edge of the wedge theorem' which originated through the challenges of relativistic quantum field theory and the theory of dispersion relations for scattering amplitudes (see [1]). In quantum field theory the important fact that the Wightman functions are holomorphic in the region of all totally space-like points is shown by a simple application of the edge of the wedge theorem.

The statements in these various versions of the edge of the wedge theorem assert the extendability of holomorphic functions defined in wedges in complex space $\mathbb{C}^{n}$ with edge in real space $\mathbb{R}^{n}$, under certain conditions. 
Recall the original version of Bogoliubov [1]:

Theorem 1.1 (Bogoliubov). Let $C \subset \mathbb{R}^{n}$ be an open proper cone with vertex at the origin and denote by $C_{r}=C \cap B(0, r)$ the intersection of $C$ with the open ball of radius $r>0$ centered at the origin; for an open nonempty set $E \subset \mathbb{R}^{n}$ introduce the wedges $W^{ \pm}=E \pm i C_{r}$ with common edge $E$. If now $F_{1}$ is a holomorphic function on $W^{+}$and $F_{2}$ is holomorphic on $W^{-}$and if $F_{1}$ and $F_{2}$ have the same boundary values on $E$,

$$
\lim _{\substack{y \rightarrow 0 \\ y \in C_{r}}} F_{1}(x+i y)=\lim _{\substack{y \rightarrow 0 \\ y \in C_{r}}} F_{2}(x-i y), \quad x \in E,
$$

then $F_{1}$ and $F_{2}$ can be extended holomorphically to a complex neighborhood $\Omega$ of $W^{+} \cup E \cup W^{-}$.

Naturally, in (1.1) it is important to specify in which sense the boundary values are considered. In Bogoliubov's version these boundary values are taken in the sense of Schwartz distributions. Note that for $n=1$ and when the boundary values are taken in the sense of continuous functions, this result is easily proven by using Morera's theorem.

Over the last fifty years, this result has been extended in several directions:

- the type of generalized functions for the boundary values in (1.1), e. g., Schwartz distributions, Sato hyperfunctions, Fourier hyperfunctions, and ultradistributions;

- the number $m$ of wedges, $m>2$;

- the 'topological nature' of the edge $E$, e. g., an open nonempty set or a maximal real submanifold.

We comment here on the case of Fourier hyperfunctions. A Fourier hyperfunction $f$ has two realizations. One is as a dual element of the test-function space $\underset{\sim}{\mathcal{O}}\left(\boldsymbol{D}^{n}\right)$ and the other is as a formal sum

$$
f(x)=\sum_{j=1}^{m} F_{j}(z)
$$

where $F_{j}(z)$ is holomorphic in a wedge $W_{j}=\boldsymbol{D}^{n}+i \Gamma_{j}$, that is, an element of the relative Cech cohomology group $H^{n}\left(\mathfrak{W}, \mathfrak{W}^{\prime} ; \tilde{\mathcal{O}}\right)$ which is isomorphic to $H_{D^{n}}^{n}\left(\boldsymbol{Q}^{n} ; \tilde{\mathcal{O}}\right)$ for a suitable relative covering $\left(\mathfrak{W}, \mathfrak{W}^{\prime}\right)$ of the pair $\left(\boldsymbol{Q}^{n}, \boldsymbol{Q}^{n} \backslash \boldsymbol{D}^{n}\right)$, where $\tilde{\mathcal{O}}$ is the sheaf of slowly increasing holomorphic functions on $\boldsymbol{Q}^{n}=\boldsymbol{D}^{n}+i \mathbb{R}^{n}$ and $\boldsymbol{D}^{n}=\mathbb{R}^{n} \cup S_{\infty}^{n-1}$ is the radial compactification of $\mathbb{R}^{n}$ (see [11, 2]). In the case of hyperfunctions and Fourier hyperfunctions, the edge of the wedge theorem tells us when the above sum is zero. Note that $H^{n}\left(\mathfrak{W}, \mathfrak{W}^{\prime} ; \tilde{\mathcal{O}}\right)$ can be expressed as the following quotient space (see [10])

$$
\oplus_{j=1}^{m} \tilde{\mathcal{O}}\left(W_{j}\right) /\left[\oplus_{j<k} \tilde{\mathcal{O}}\left(W_{j}+W_{k}\right)\right] .
$$


This denominator appears in the general edge of the wedge theorem for Fourier hyperfunctions (see [22]).

Remark 1.2. Relation (1.2) 'contains' the most important versions of the EOW: If $\sum_{j=1}^{m} F_{j}(z)=f(x)=0$, then there exist functions $H_{j k} \in$ $\tilde{\mathcal{O}}\left(W_{j}+W_{k}\right)$ for $j<k$ such that

$$
F_{j}(z)=\sum_{k=1}^{m} H_{j k}(z), j=1, \ldots, m
$$

where we put

$$
H_{j k}(z)=-H_{k j}(z) \text { for } j>k \text { and } H_{j j}(z)=0 .
$$

The above statement is Martineau's version of the EOW theorem [12, 13, 14.

Note that conversely, for the functions $F_{j}$ defined by (1.3) with functions $H_{j k}$ satisfying relation (1.4), the sum

$$
\sum_{j=1}^{m} F_{j}(z)=\sum_{j=1}^{m} \sum_{k=1}^{m} H_{j k}(z)
$$

is reduced to zero and defines the zero Fourier hyperfunction.

When $m=2$, we have Epstein's version of the EOW theorem [5], i.e., if $F_{j} \in \tilde{\mathcal{O}}\left(W_{j}\right)(j=1,2)$ define the same Fourier hyperfunction, then $F_{j} \in \tilde{\mathcal{O}}\left(W_{j}\right)(j=1,2)$ are analytically continued to $H_{12} \in \tilde{\mathcal{O}}\left(W_{1}+\right.$ $W_{2}$ ). (Epstein's original version is formulated in terms of Schwartz distributions as boundary values).

If $\Gamma_{2}=-\Gamma_{1}$ and if the boundary values are taken in the sense of Schwartz distributions, then we have Bogoliubov's version of the EOW theorem.

In our recent investigations of relativistic quantum field theory with a fundamental length (see [3, 4, 20, 21]) we need a version of the edge of the wedge theorem for tempered ultra-hyperfunctions and it is this version which is treated in this article.

\section{Preliminaries}

2.1. Global and local versions of the EOW Theorem. In these preliminary considerations we put the global and the local forms of the edge of the wedge theorems for hyperfunctions and ultra-hyperfunctions into the perspective of cohomology theory.

Let us recall the global form of this theorem for hyperfunctions (For simplicity and our intended application to quantum field theory, we consider in this paper only Bogoliubov's version for $\Gamma=V_{+}$):

Let $V_{+} \subset \mathbb{R}^{4}$ denote the forward light-cone; suppose that $F_{1}$ is an analytic function in $T\left(V_{+}\right)=\mathbb{R}^{4}+i V_{+}$and $F_{2}$ an analytic function in 
$T\left(-V_{+}\right)$. Then the two functions $F_{i}(i=1,2)$ define hyperfunctions $f_{i}$ on $\mathbb{R}^{4}$. If $f_{1}=f_{2}$, then $F_{i}$ are analytically continued to an entire function $F$.

The local form of the EOW theorem for hyperfunction can be formulated as follows. Let $U$ be an open set in $\mathbb{R}^{n}$ and $V$ an open set in $\mathbb{C}^{n}$ such that $U=V \cap \mathbb{R}^{n}$. Then we have the canonical restriction map

$$
H_{\mathbb{R}^{n}}^{n}\left(\mathbb{C}^{n}, \mathcal{O}\right) \rightarrow H_{U}^{n}(V, \mathcal{O})=H^{n-1}(V \backslash U, \mathcal{O}),
$$

where $\mathcal{O}$ is the sheaf of holomorphic functions on $\mathbb{C}^{n} . H_{U}^{n}(V, \mathcal{O})$ is independent of the complex neighborhood $V$ of $U$ by the excision theorem, and the presheaf $\left\{U \rightarrow H_{U}^{n}(V, \mathcal{O})\right\}$ is the sheaf of hyperfunctions on $\mathbb{R}^{n}$ which is often denoted by $\mathcal{B}$. The local form of the EOW theorem now reads (we use the notation from above):

If $f_{1}=f_{2}$ in $U$ (or the restrictions $f_{j \mid U}(j=1,2)$ coincide or the support of $f_{1}-f_{2}$ is contained in the complement of $U$ ), then $F_{j}$ are analytically continued to each other through $U$.

There are two ways to treat the EOW theorem; the functional method (see [9]) and the cohomological method (see [17, 10]). The functional method uses the notion of the analytic wave front set $W F_{a}(f)$ of hyperfunctions $f$ on $\mathbb{R}^{n} \times\left(\mathbb{R}^{n} \backslash\{0\}\right)$ and the decomposition of $W F_{a}(f)$. The colomological method uses the notion of the flabby sheaf $\mathcal{C}$ of micro functions on $\mathbb{R}^{n} \times S^{n-1}$ and the exact sequence

$$
0 \rightarrow \mathcal{A} \rightarrow \mathcal{B} \rightarrow \pi_{*} \mathcal{C} \rightarrow 0,
$$

where $\mathcal{A}$ is the sheaf of real analytic functions on $\mathbb{R}^{n}$ and $\pi_{*} \mathcal{C}$ is the direct image of $\mathcal{C}$ under the projection $\pi: \mathbb{R}^{n} \times S^{n-1} \rightarrow \mathbb{R}^{n}$, i.e., the sheaf on $\mathbb{R}^{n}$ defined by the correspondence

$$
\mathbb{R}^{n} \supset U \rightarrow \mathcal{C}\left(\pi^{-1}(U)\right) .
$$

Finally we comment on the difficulties for the EOW for tempered ultrahyperfunctions.

Any element of $\mathcal{T}\left(T\left(\mathbb{R}^{n}\right)\right)^{\prime}$ belongs to some $\mathcal{T}(T(K))^{\prime}, K=[-k, k]^{n}$ for suitable $k>0$ and

$$
\mathcal{T}(T(K))^{\prime}=H^{n}\left(\mathfrak{W}, \mathfrak{W}^{\prime} ; \mathcal{O}_{0}\right),
$$

where $\mathfrak{W}=\mathfrak{W}^{\prime} \cup\left\{\mathbb{C}^{n}\right\}$ and $\mathfrak{W}^{\prime}=\left\{T\left(E_{j}\right) ; j=1, \ldots, n\right\}, E_{j}=\{y \in$ $\left.\mathbb{R}^{n} ;\left|y_{j}\right|>k\right\}$ are relative covering of $\left(\mathbb{C}^{n}, \mathbb{C}^{n} \backslash T(K)\right.$ (see [ㅇ, [18]). Hyperfunctions are localized in a relatively open set $U$ of the closed set $\mathbb{R}^{n}$ in $\mathbb{C}^{n}$ by the formula (2.1). On the other hand, tempered ultrahyperfunctions may be localized in a relatively open set $U$ of the closed set $T(K)$ of $\mathbb{C}^{n}$, but not in a relatively open set $U$ of the closed set $\mathbb{R}^{n}$ in $\mathbb{C}^{n}$. This is the reason why tempered ultra-hyperfunctions have no (standard) localization property in $\mathbb{R}^{n}$. In [3, 20, 4, this property has been successfully applied to axiomatic quantum field theory in order to formulate such a theory with a fundamental length. 
The global form of the edge of the wedge theorem for tempered ultra-hyperfunctions reads: Let $\Gamma=\left\{y \in \mathbb{R}^{n} ; y_{j}>k, j=1, \ldots, n\right\}$. If $F_{1}$ is a polynomially increasing holomorphic function in $T(\Gamma)$ and $F_{2}$ a polynomially increasing holomorphic function in $T(-\Gamma)$, then these functions $F_{i}(i=1,2)$ define tempered ultra-hyperfunctions $f_{i}$ and if $f_{1}=f_{2}$, then $F_{i}$ are analytically continued to a polynomial $F$ (see [18]).

Remark 2.1. It is interesting to note that even though $T(\Gamma)$ and $T(-\Gamma)$ are separated by a gap of size $2 \sqrt{n} k$ the functions $F_{1}$ and $F_{2}$ are analytically continued to each other!

At first sight this seems to be quite surprising. However, from the point of view of the cohomological definition of ultra-hyperfunctions, this result is not so surprising, since $H^{n}\left(\mathfrak{W}, \mathfrak{W}^{\prime} ; \mathcal{O}_{0}\right)$ has the following representation

$$
\mathcal{O}_{0}\left(\mathbb{C}^{n} \# T(K)\right) / \sum_{j=1}^{n} \mathcal{O}_{0}\left(W_{\hat{j}}\right)
$$

where

$$
\begin{gathered}
\left.\mathbb{C}^{n} \# T(K)\right)=T\left(E_{1}\right) \cap \cdots \cap T\left(E_{n}\right), \\
W_{\hat{j}}=T\left(E_{1}\right) \cap \cdots \cap \widehat{T\left(E_{j}\right)} \cap \cdots \cap T\left(E_{n}\right)
\end{gathered}
$$

and the denominator of this representation then shows this result. We explain this in more detail in the next subsection for the one dimensional case. The cohomological treatment of ultra-hyperfunctions is given in [15, 16] and the above representation was presented in [18. The global form of the EOW theorem for tempered ultra-hyperfunctions has been shown in [24] and some preliminary version in [6, 7].

In the functional method [9], Hörmander used a kernel $K(z)$ defined by

$$
K(z)=(2 \pi)^{-n} \int e^{i\langle z, \xi\rangle} / I(\xi) d \xi, I(\xi)=\int_{|\omega|=1} e^{-\langle\omega, \xi\rangle} d \omega
$$

to prove the edge of the wedge theorem. For the proof of the local form of the EOW theorem for tempered ultra-hyperfunctions we also use the functional method with some modification $K_{r}(z)=r^{-n} K(z / r)$ of this kernel $K(z)$ for $r>0$.

The local form of the edge of the wedge theorem for hyperfunction has the following formulation $\left(F_{j}\right.$ and $f_{j}$ are related as in the above results): If $f_{1}=f_{2}$ in an open set $O$, then $F_{1}$ and $F_{2}$ are analytically continued to each other through $O$.

Since tempered ultra-hyperfunctions have no localization property, it is not easy to formulate a local form of the edge of the wedge theorem. In this paper, we suggest a formulation of the local version of the edge of the wedge theorem by using the notion of a carrier. 
Remark 2.2. For the one dimensional case, the Cauchy-Hilbert transformation (2.6) gives the isomorphism of the space $\mathcal{O}(L)^{\prime}$ of analytic functionals with carriers in $L$ onto the relative cohomology group of covering (2.7) of the pair $(\mathbb{C}, \mathbb{C} \backslash L)$. But for the multi dimensional case, we need the additional assumption to have the expression of the space $\mathcal{O}(L)^{\prime}$ of analytic functionals with carriers in a compact set $L \subset \mathbb{C}^{n}$ as a collection of holomorphic functions. In fact, the isomorphism of the space $\mathcal{O}(L)^{\prime}$ of analytic functionals with carriers in a compact set $L \subset \mathbb{C}^{n}$ onto the relative cohomology group $H_{L}^{n}\left(\mathbb{C}^{n}, \mathcal{O}\right)$ is proven under the condition a) $H^{p}(L, \mathcal{O})=0$ for $p=1,2, \ldots$ (see [18), and the isomorphism of $H_{L}^{n}\left(\mathbb{C}^{n}, \mathcal{O}\right)$ onto the cohomology group $H^{n}\left(\mathfrak{W}, \mathfrak{W}^{\prime} ; \mathcal{O}\right)$ of a covering $\left(\mathfrak{W}, \mathfrak{W}^{\prime}\right)$ of the pair $\left(\mathbb{C}^{n}, \mathbb{C}^{n} \backslash L\right)$ is proven under the condition b) $H^{p}\left(W_{\lambda_{0}} \cap \ldots \cap W_{\lambda_{m}}\right)=0$ for $p \geq 1$ and $W_{\lambda_{0}}, \ldots, W_{\lambda_{m}} \in \mathfrak{W}$ (Leray theorem, see [19]). In the case of hyperfunctions, a compact set $L \subset \mathbb{R}^{n}$ satisfies condition a) and there exists a relative covering $\left(\mathfrak{W}, \mathfrak{W}^{\prime}\right)$ of the pair $\left(\mathbb{C}^{n}, \mathbb{C}^{n} \backslash L\right)$ which satisfies condition b) (see [10, 19]). But in the case of analytic functional $\mathcal{O}(L)^{\prime}$, conditions a) and b) may not necessarily be satisfied for some compact set $L \subset \mathbb{C}^{n}$ and any relative covering $\left(\mathfrak{W}, \mathfrak{W}^{\prime}\right)$ of the pair $\left(\mathbb{C}^{n}, \mathbb{C}^{n} \backslash L\right)$. Therefore, in this paper we employ the functional method.

The main result in this regard is Corollary 4.3 which has an intimate connection to axiomatic quantum field theory with a fundamental length (see [21]). This corollary says that if $f_{1}-f_{2} \in \mathcal{T}(L)^{\prime}$ for some $\ell$-neighborhood

$$
L=\left\{w \in \mathbb{C}^{4} ; \exists x \in V|\operatorname{Re} w-x|+|\operatorname{Im} w|_{1}<\ell\right\},
$$

of the light-cone $V, \ell>0$, then $F_{i}(i=1,2)$ are analytically continued to each other through a set

$$
\left\{x \in \mathbb{R}^{4} ; \operatorname{dist}(x, V)>(\sqrt{2}+1) \ell\right\} .
$$

2.2. The one dimensional case. In order to explain the basic idea of our strategy of proof for the local version of the EOW theorem for tempered ultrahyperfunctions, we illustrate it here for the technically much simpler case of one dimension. And we prepare this with explaining the proof for hyperfunctions in one variable.

The space of hyperfunctions of one variable is the quotient space

$$
\mathcal{B}(\mathbb{R})=\mathcal{O}(\mathbb{C} \backslash \mathbb{R}) / \mathcal{O}(\mathbb{C}) .
$$

Let $F_{1}$ (resp. $F_{2}$ ) be a holomorphic function in the upper (resp. lower) half plane. Then the pair of functions $\left(F_{1}, F_{2}\right) \in \mathcal{O}(\mathbb{C} \backslash \mathbb{R})$ defines an element $f\left(=F_{1}-F_{2}\right)$ of $\mathcal{B}(\mathbb{R})$. If $f=0\left(F_{1}=F_{2}\right)$, then $\left(F_{1}, F_{2}\right) \in$ $\mathcal{O}(\mathbb{C})$. This shows that $F_{1}, F_{2}$ coincide with an element $F$ of $\mathcal{O}(\mathbb{C})$. Thus the EOW theorem automatically follows from the cohomological definition of hyperfunctions. 
The local version of the EOW theorem for hyperfunctions is also a direct consequence of the cohomological definition of hyperfunctions on an open set $U$ of $\mathbb{R}$ :

$$
\mathcal{B}(U)=\mathcal{O}(V \backslash U) / \mathcal{O}(V),
$$

where $V$ is a complex neighborhood of $U$ such that $U=V \cap \mathbb{R}$.

Now consider the case of tempered ultra-hyperfunctions. Denote $K=[-k, k]$ for $k>0$ and $T(K)=\{z \in \mathbb{C} ;|\operatorname{Im} z| \leq k\}$. Note that formula (2.2) shows that any tempered ultra-hyperfunction $f \in \mathcal{T}(T(\mathbb{R}))^{\prime}$ can be expressed as an element of the space

$$
H_{T(K)}^{1}\left(\mathbb{C}, \mathcal{O}_{0}\right) \cong \mathcal{O}_{0}(\mathbb{C} \backslash T(K)) / \mathcal{O}_{0}(\mathbb{C})
$$

for some $k>0$. Let $F_{1}$ (resp. $F_{2}$ ) be a polynomially increasing holomorphic function in $\{z \in \mathbb{C} ; \operatorname{Im} z>k\}$ (resp. $\{z \in \mathbb{C} ;-\operatorname{Im} z>k\}$ ). Then the pair of functions $\left(F_{1}, F_{2}\right) \in \mathcal{O}_{0}(\mathbb{C} \backslash T(K))$ defines an element $f$ of $H_{T(K)}^{1}\left(\mathbb{C}, \mathcal{O}_{0}\right)$. If $f=0$, then $\left(F_{1}, F_{2}\right) \in \mathcal{O}_{0}(\mathbb{C})$. This shows that $F_{1}, F_{2}$ coincide with an element $F$ of $\mathcal{O}_{0}(\mathbb{C})$. Thus the global form of the EOW theorem for tempered ultra-hyperfunctions automatically follows from the cohomological definition of tempered ultra-hyperfunctions.

Since the notion of localization for ultra-hyperfunctions is not available in the above sense, there is no literature about the local version of the EOW theorem for ultra-hyperfunctions. The notion of localization for generalized functions has an intimate connection with the notion of support. However, ultra-hyperfunctions have no supports in general, but they are a special kind of analytic functionals and have carriers.

Definition 2.3. Let $L$ be a compact set in $\mathbb{C}$. $L$ is called a carrier of an analytic functional $f$ (a continuous linear functional on the space $\mathcal{O}(\mathbb{C})$ of entire functions), if $f$ satisfies

$$
|f(\phi)| \leq C_{V} \sup _{z \in V}|\phi(z)|
$$

for any open neighborhood $V$ of $L$.

Let $K=[a, b] \subset \mathbb{R}$ and $H_{K}^{1}(\mathbb{C}, \mathcal{O})$ be the space of hyperfunctions with supports in $K$, which is isomorphic to the space of analytic functionals with carriers in $K$ :

$$
H_{K}^{1}(\mathbb{C}, \mathcal{O}) \cong \mathcal{O}(\mathbb{C} \backslash K) / \mathcal{O}(\mathbb{C}) .
$$

Every $F \in \mathcal{O}(\mathbb{C} \backslash K)$ defines a functional on the space of functions $\phi$ which are holomorphic in a complex neighborhood $V$ of $K$ by the formula

$$
\phi \rightarrow f(\phi)=-\int_{C} F(z) \phi(z) d z,
$$

where $C$ is a closed path that encircles $K$ once in the positive direction. 
Let $E_{\xi}^{t}(z)=(4 \pi t)^{-1 / 2} e^{-(z-\xi)^{2} / 4 t}$ for $\xi \in \mathbb{R} \backslash K$. Clearly $E_{\xi}^{t}$ is holomorphic in a complex neighborhood $V$ of $K$ and, as $t \rightarrow 0, E_{\xi}^{t} \rightarrow \delta(x-\xi)$ and one has the estimate

$$
\left|f\left(E_{\xi}^{t}\right)\right| \leq M_{C} \sup _{x+i y \in C}(4 \pi t)^{-1 / 2} e^{\left(y^{2}-(x-\xi)^{2}\right) / 4 t} \rightarrow 0
$$

as $t \rightarrow 0+$, since $C$ can be chosen arbitrary close to $K$.

Let $L=[a, b]+i[-\ell, \ell]$ and $f$ be an analytic functional with carrier $L$. Then the Cauchy-Hilbert transformation $F$ of $f$ is defined by

$$
F(z)=(2 \pi i)^{-1} f_{w}(1 /(w-z)) ;
$$

it is an element of

$$
H_{L}^{1}\left(\mathbb{C}, \mathcal{O}_{0}\right) \cong \mathcal{O}_{0}(\mathbb{C} \backslash L) / \mathcal{O}_{0}(\mathbb{C}),
$$

i.e., the space of tempered ultra-hyperfunctions with carriers in $L$, and it reproduces the functional $f$ by formula (2.4) with a closed path $C$ that encircles $L$, i.e.,

$$
-\int_{C} F(z) \phi(z) d z=f_{w}\left((2 \pi i)^{-1} \int_{C} \phi(z) /(z-w) d z\right)=f(\phi) .
$$

For a tempered ultra-hyperfunction $f$ with carrier $L$, we have (2.5) if $\xi \in \mathbb{R} \backslash[a-\ell, b+\ell]$. This means that $\xi$ is considered to be outside of $[a, b]$ only if $\xi \in \mathbb{R} \backslash[a-\ell, b+\ell]$ !

Remark 2.4. Heuristically we read this fact as follows:

An ultra-hyperfunction $f$ becomes 'aware' of $\xi$ being outside of $[a, b]$ only if $\xi \in \mathbb{R} \backslash[a-\ell, b+\ell]$, while a hyperfunction can be 'aware' of $\xi$ being outside of $[a, b]$ if $\xi \in \mathbb{R} \backslash[a, b]$.

This difference can also be understood through cohomological considerations. Let $U$ be an open set in $\mathbb{R}$ such that $[a, b] \cap U=\emptyset$ and $U=V \cap \mathbb{R}$ for an open set $V$ in $\mathbb{C}$. In the case of hyperfunctions, we have the restriction

$$
H_{K}^{1}(\mathbb{C}, \mathcal{O}) \rightarrow H_{U}^{1}(V, \mathcal{O}) \cong \mathcal{O}(V \backslash K) / \mathcal{O}(V)=\mathcal{O}(V) / \mathcal{O}(V)=0 .
$$

But for the case of ultra-hyperfunctions, we have

$$
H_{L}^{1}\left(\mathbb{C}, \mathcal{O}_{0}\right) \rightarrow H_{U}^{1}\left(V, \mathcal{O}_{0}\right) \cong \mathcal{O}_{0}(V \backslash L) / \mathcal{O}_{0}(V)
$$

and there exists an open set $V$ in $\mathbb{C}$ satisfying $[a, b] \cap U=\emptyset$ and $U=V \cap \mathbb{R}$ but $V \backslash L \neq V$ and $\mathcal{O}_{0}(V \backslash L) / \mathcal{O}_{0}(V) \neq 0$.

Now we study the EOW theorem by using Hörmander's kernel $K(z)$. First, recall that Dirac's $\delta$ function can be expressed as follows:

$$
\begin{gathered}
\delta(x)=(2 \pi)^{-1} \int_{-\infty}^{\infty} e^{i \xi x} d \xi \\
=(2 \pi)^{-1} \lim _{\epsilon \rightarrow 0} \int_{-\infty}^{\infty} e^{i \xi(x+i \epsilon)} \frac{e^{\xi}}{e^{\xi}+e^{-\xi}} d \xi+(2 \pi)^{-1} \lim _{\epsilon \rightarrow 0} \int_{-\infty}^{\infty} e^{i \xi(x-i \epsilon)} \frac{e^{-\xi}}{e^{\xi}+e^{-\xi}} d \xi
\end{gathered}
$$




$$
\begin{gathered}
=(2 \pi)^{-1} \lim _{\epsilon \rightarrow 0} \int_{-\infty}^{\infty} \frac{e^{i \xi(x+i \epsilon-i)}}{e^{\xi}+e^{-\xi}} d \xi+(2 \pi)^{-1} \lim _{\epsilon \rightarrow 0} \int_{-\infty}^{\infty} \frac{e^{i \xi(x-i \epsilon+i)}}{e^{\xi}+e^{-\xi}} d \xi \\
=(2 \pi)^{-1} \lim _{\epsilon \rightarrow 0} \sum_{\omega= \pm 1} \int_{-\infty}^{\infty} \frac{e^{i \xi(x+i(1-\epsilon) \omega)}}{e^{\xi}+e^{-\xi}} d \xi .
\end{gathered}
$$

Now introduce the function $K(z)$ of (2.3) for $n=1$ :

$$
K(z)=(2 \pi)^{-1} \int_{-\infty}^{\infty} e^{i \xi z} \frac{1}{2} \operatorname{sech} \xi d \xi=\frac{1}{4} \operatorname{sech}(\pi z / 2)
$$

for $\operatorname{sech} \xi=\frac{2}{e^{\xi}+e^{-\xi}}$. Then the above representation of the Dirac's delta function can be rewritten as

$$
\begin{gathered}
\delta(x)=\lim _{\epsilon \rightarrow+0}[K(x+i \epsilon-i)+K(x-i \epsilon+i)] \\
=\lim _{\epsilon \rightarrow+0}(1 / 4)[\operatorname{sech} \pi(x+i \epsilon-i) / 2+\operatorname{sech} \pi(x-i \epsilon+i) / 2] \\
=\lim _{\epsilon \rightarrow+0}(1 / 4) i[\operatorname{cosech} \pi(x+i \epsilon) / 2-\operatorname{cosech} \pi(x-i \epsilon) / 2],
\end{gathered}
$$

where $\operatorname{cosech} \xi=\frac{2}{e^{\xi}-e^{-\xi}}$. Since the difference between $(1 / 4) i \operatorname{cosech} \pi z / 2$ and $-1 /(2 \pi i z)$ is holomorphic function in $\{z \in \mathbb{C} ;|\operatorname{Im} z|<1\}$, formula (2.8) is equivalent to the famous formula

$$
\delta(x)=-(2 \pi i)^{-1} \lim _{\epsilon \rightarrow+0}[1 /(x+i \epsilon)-1 /(x-i \epsilon)] .
$$

The singular points of $\operatorname{sech} \xi$ are $\xi=i(1+2 n) \pi / 2 n=0, \pm 1, \pm 2, \ldots$ and those of $K(z)$ are $z=i(1+2 n) n=0, \pm 1, \pm 2, \ldots$. Therefore we have, for $\phi \in \mathcal{T}(T(\mathbb{R}))$ and $0<R \leq 1$

$$
\begin{aligned}
\phi(0) & =\lim _{\epsilon \rightarrow+0} \int[K(x+i \epsilon-i)+K(x-i \epsilon+i)] \phi(x) d x \\
& =\lim _{\epsilon \rightarrow+0} \sum_{\omega= \pm 1} \int K(x-i \omega \epsilon+i \omega) \phi(x) d x \\
& =\lim _{\epsilon \rightarrow+0} \sum_{\omega= \pm 1} \int K(x-i R \omega-i \omega \epsilon+i \omega) \phi(x-i R \omega) d x \\
& =\sum_{\omega= \pm 1} \int K(x-i \omega R+i \omega) \phi(x-i R \omega) d x
\end{aligned}
$$

Let $K_{r}(z)=r^{-1} K(z / r)$. Then we can reformulate the above relation as

$$
\delta(x)=\lim _{\epsilon \rightarrow+0}\left[K_{r}(x+i r \epsilon-i r)+K_{r}(x-i r \epsilon+i r)\right]
$$

and for $1<R \leq r$

$$
\sum_{\omega= \pm 1} \int_{-\infty}^{\infty} K_{r}(x+i(r-R) \omega) \phi(x-i R \omega) d x=\phi(0)
$$


and

$$
\begin{aligned}
& \sum_{\omega= \pm 1} \int_{-\infty}^{\infty} K_{r}(x-t+i(r-R) \omega) \phi(x-i R \omega) d x \\
= & \sum_{\omega= \pm 1} \int_{-\infty}^{\infty} K_{r}(x+i(r-R) \omega) \phi(x+t-i R \omega) d x=\phi(t) .
\end{aligned}
$$

Let $\Gamma_{1}=\{y \in \mathbb{R} ; y>\ell\}$ and $\Gamma_{2}=\{y \in \mathbb{R} ;-y>\ell\}$. Given $F_{j} \in$ $\mathcal{O}_{0}\left(T\left(\Gamma_{j}\right)\right)$ denote by $u_{j}$ the tempered ultra-hyperfunction defined by

$$
u_{j}(\phi)=\int_{-\infty}^{\infty} F_{j}(x+i y) \phi(x+i y) d x
$$

for $y \in \Gamma_{j}$. Choose $r>\ell$ and define

$$
U_{j}(z)=u_{j} * K_{r}(z)=\int_{-\infty}^{\infty} F_{j}(\xi+i \eta) K_{r}(z-\xi-i \eta) d \xi
$$

for $\eta \in \Gamma_{j}$. Then $U_{j}(z)$ is analytic in $V_{j}$,

$$
\begin{aligned}
& V_{1}=\cup_{\eta \in \Gamma_{1}}\{z \in \mathbb{C} ;|\operatorname{Im}(z-i \eta)|<r\}=\{z \in \mathbb{C} ; \operatorname{Im} z>\ell-r\}, \\
& V_{2}=\cup_{\eta \in \Gamma_{2}}\{z \in \mathbb{C} ;|\operatorname{Im}(z-i \eta)|<r\}=\{z \in \mathbb{C} ; \operatorname{Im} z<-\ell+r\} .
\end{aligned}
$$

Note that (2.9) implies

$$
\begin{gathered}
\sum_{\omega= \pm 1} \int_{-\infty}^{\infty} U_{j}(x+i(r-R) \omega) \phi(x-i R \omega) d x \\
=\sum_{\omega= \pm 1} \int_{-\infty}^{\infty} u_{j} * K_{r}(x+i(r-R) \omega) \phi(x-i R \omega) d x \\
=\sum_{\omega= \pm 1} \int_{-\infty}^{\infty} \int_{-\infty}^{\infty} F_{j}(\xi+i \eta) K_{r}(x-\xi-i \eta+i(r-R) \omega) \phi(x-i R \omega) d x d \xi \\
=\int_{-\infty}^{\infty} F_{j}(\xi+i \eta) \phi(\xi+i \eta) d \xi=u_{j}(\phi) .
\end{gathered}
$$

If $u_{1}=u_{2}$, then $U_{1}(z)=U_{2}(z)$ in $V_{1} \cap V_{2}=\{z \in \mathbb{C} ;|\operatorname{Im} z|<r-\ell\}$, and the two functions $U_{1}, U_{2}$ are continued to a function $U$ which is analytic in $V_{1} \cup V_{2}=\mathbb{C}$.

Now introduce the function

$$
H(z)=\sum_{\omega= \pm 1} U(z+i r \omega)
$$

clearly $H$ is an entire function, and we have for $y \in \Gamma_{1}, \phi \in \mathcal{T}(T(\mathbb{R}))$, and $\ell-r \leq \sigma<\ell$

$$
\begin{gathered}
\int_{-\infty}^{\infty} H(x+i y) \phi(x+i y) d x=\sum_{\omega= \pm 1} \int_{-\infty}^{\infty} U(x+i y+i r \omega) \phi(x+i y) d x \\
=\sum_{\omega= \pm 1} \int_{-\infty}^{\infty} U(x+i(r-\ell+\sigma) \omega) \phi(x-i(\ell-\sigma) \omega) d x
\end{gathered}
$$




$$
\begin{gathered}
=\sum_{\omega= \pm 1} \int_{-\infty}^{\infty} U_{1}(x+i(r-\ell+\sigma) \omega) \phi(x-i(\ell-\sigma) \omega) d x \\
=u_{1}(\phi)=\int_{-\infty}^{\infty} F_{1}(x+i y) \phi(x+i y) d x
\end{gathered}
$$

where we used the fact that $U(z)=U_{1}(z)$ for $\operatorname{Im} z>\ell-r$ and relation (2.10), hence

$$
\int_{-\infty}^{\infty} H(x+i y) \phi(x+i y) d x=\int_{-\infty}^{\infty} F_{1}(x+i y) \phi(x+i y) d x
$$

for $\phi \in \mathcal{T}(T(\mathbb{R}))$. This shows that $H(z)=F_{1}(z)$ in $T\left(\Gamma_{1}\right)$, and in the same way we get $H(z)=F_{2}(z)$ in $T\left(\Gamma_{2}\right)$. This proves the global form of the EOW theorem for tempered ultra-hyperfunctions of one variable.

Next we discuss the local version. To this end assume that the carrier of $u_{1}-u_{2}$ is contained in $L=[a, b]+i[-\ell, \ell]$, instead of $u_{1}=u_{2}$. This will lead to the local form of the EOW theorem.

Introduce the function $U_{12}$ by

$$
U_{12}(z)=\left(u_{1}-u_{2}\right) * K_{r}(z)
$$

Since the singular points of $K_{r}(z)$ are $z=i(1+2 n) r, n=0, \pm 1, \pm 2, \ldots$, $K_{r}(z-w)$, for $\operatorname{Re} z \notin[a, b]$, is holomorphic in a neighborhood of $L$ and $U_{12}(z)$ is holomorphic in $Z=\{z \in \mathbb{C} ; \operatorname{Re} z \notin[a, b]\}$. Since $u_{1}=$ $u_{2}+u_{1}-u_{2}$,

$$
U_{1}(z)=U_{2}(z)+U_{12}(z)
$$

$U_{1}(z)$ is holomorphic in $V_{1}$ and $U_{2}(z)+U_{12}(z)$ is holomorphic in $Z \cap V_{2}$. Therefore, in the same way as in the global case, $U_{1}(z)$ can be analytically continued to a function $U_{1}^{\prime}(z)$ which is holomorphic in $Z$ and $F_{1}(z)$ can be analytically continued to $H_{1}(z)=\sum_{\omega= \pm 1} U_{1}^{\prime}(z+i r \omega)$ which is holomorphic in $Z$. In the same way, $F_{2}(z)$ can be analytically continued to $H_{2}(z)$ which is holomorphic in $Z$. In order to show $H_{1}(z)=H_{2}(z)$ in $Z$, we introduce a path $C=C_{1}+\ldots+C_{5}$ consisting of line segments $C_{1}=(-\infty, a-2 \ell], C_{2}=[a-2 \ell, a+i 2 \ell]$, $C_{3}=[a+i 2 \ell, b+i 2 \ell], C_{4}=[b+i 2 \ell, b+2 \ell]$ and $C_{5}=[b+2 \ell, \infty)$ (see Figure 1). Let $E_{\xi}^{t}(z)=(4 \pi t)^{-1 / 2} e^{-(\xi-z)^{2} / 4 t}$ and $\xi \in \mathbb{R}$ such that

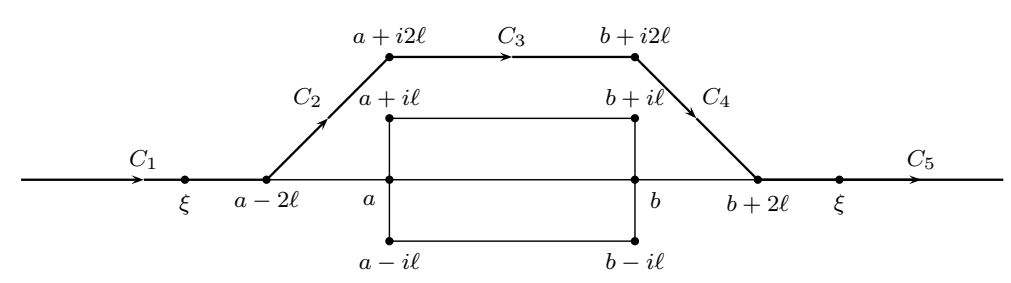

Figure 1. The path of integration $C=C_{1}+C_{2}+C_{3}+$ $C_{4}+C_{5}$ 
$\xi<a-2 \ell$ or $\xi>b+2 \ell$. Then we have

$$
\begin{gathered}
u_{1}\left(E_{\xi}^{t}\right)=\int_{-\infty}^{\infty} F_{1}(x+i 2 \ell) E_{\xi}^{t}(x+i 2 \ell) d x \\
=\int_{-\infty}^{\infty} H_{1}(x+i 2 \ell) E_{\xi}^{t}(x+i 2 \ell) d x=\int_{C} H_{1}(z) E_{\xi}^{t}(z) d z \rightarrow H_{1}(\xi)
\end{gathered}
$$

as $t \rightarrow 0+$. Here we used the fact that if $|\xi-\operatorname{Re} z|>|\operatorname{Im} z|,\left|E_{\xi}^{t}(z)\right|=$ $(4 \pi t)^{-1} e^{-\left(|\xi-\operatorname{Re} z|^{2}-|\operatorname{Im} z|^{2}\right) / 4 t} \rightarrow 0$ as $t \rightarrow 0+$ and if $z \in \mathbb{R}, E_{\xi}^{t}(z) \rightarrow$ $\delta(z-\xi)$, i.e., $E_{\xi}^{t}(z) \rightarrow 0$ for $z \in C_{2}+C_{3}+C_{4}$, and if $\xi \in C_{1}$ (resp. $\left.\xi \in C_{5}\right)$ then $E_{\xi}^{t}(z) \rightarrow \delta(z-\xi)$ and $E_{\xi}^{t}(z) \rightarrow 0$ for $z \in C_{5}$ (resp. $z \in C_{1}$ ). If we choose a curve $C^{\prime}=C_{1}^{\prime}+\ldots+C_{5}^{\prime}$, where $C_{1}^{\prime}=(-\infty, a-2 \ell]$, $C_{2}^{\prime}=[a-2 \ell, a-i 2 \ell], C_{3}^{\prime}=[a-i 2 \ell, b-i 2 \ell], C_{4}^{\prime}=[b-i 2 \ell, b+2 \ell]$, $C_{5}^{\prime}=[b+2 \ell, \infty)$, then we have

$u_{2}\left(E_{\xi}^{t}\right)=\int_{-\infty}^{\infty} H_{2}(x-i 2 \ell) E_{\xi}^{t}(x-i 2 \ell) d x=\int_{C^{\prime}} H_{2}(z) E_{\xi}^{t}(z) d z \rightarrow H_{2}(\xi)$

as $t \rightarrow 0+$. Moreover, we find

$$
\left|u_{1}\left(E_{\xi}^{t}\right)-u_{2}\left(E_{\xi}^{t}\right)\right| \leq M_{V} \sup _{z \in V}\left|E_{\xi}^{t}(z)\right| \rightarrow 0
$$

as $t \rightarrow 0+$ for some neighborhood $V$ of $L$. Thus $H_{1}(\xi)=H_{2}(\xi)$ follows and consequently there exists a function $H$ which is holomorphic in $\mathbb{C} \backslash L$ and which is the common extension of $F_{1}$ and $F_{2}$. Therefore $F_{1}$ and $F_{2}$ have the common extension $H \in \mathcal{O}_{0}(\mathbb{C} \backslash L)$, and $H$ is an element of $H_{L}^{1}\left(\mathbb{C}, \mathcal{O}_{0}\right)$ of (2.7).

It is interesting to note that the cohomology group $H_{L}^{1}\left(\mathbb{C}, \mathcal{O}_{0}\right)$ of tempered ultra-hyperfunctions with carriers in $L$ appears again here in the argument of EOW theorem using the kernel $K_{r}(z)$.

\section{Global edge of the Wedge theorem}

In this section we prove the global version of EOW theorem in higher dimension using the functional method. This will help in understanding the proof of the local version.

First we recall the test-function space $\mathcal{T}\left(T\left(\mathbb{R}^{n}\right)\right)$ of tempered ultrahyperfunction. Let $T(A)=\mathbb{R}^{n}+i A$ for $A \subset \mathbb{R}^{n}$. Let $K \subset \mathbb{R}^{n}$ be a convex compact set and $\mathcal{T}_{b}(T(K))$ the set of continuous functions on $T(K)$ which are holomorphic in the interior of $T(K)$ and satisfy

$$
\|f\|^{T(K), j}=\sup \left\{\left|z^{p} f(z)\right| ; z \in T(K),|p| \leq j\right\}<\infty, j=1,2, \ldots .
$$

There is a natural restriction mapping $P_{K, L}: \mathcal{T}_{b}(T(K)) \rightarrow \mathcal{T}_{b}(T(L))$ for $K \supset L$.

Definition 3.1. $\mathcal{T}\left(T\left(\mathbb{R}^{n}\right)\right)$ is the projective limit

$$
\mathcal{T}\left(T\left(\mathbb{R}^{n}\right)\right)=\lim _{\leftarrow} \mathcal{T}_{b}(T(K)), K \uparrow \mathbb{R}^{n}
$$


of the projective system $\left(\mathcal{T}_{b}(T(K)), P_{K, L}\right)$, where $K$ runs through the convex compact sets in $\mathbb{R}^{n}$.

It is known that the kernel $K(z)$ of $(2.3)$ is a rapidly decreasing holomorphic function in $\left\{z \in \mathbb{C}^{n} ;|\operatorname{Im} z|^{2}<1+|\operatorname{Re} z|^{2}\right\}$ (see [22]), and the following lemma holds (see [23]).

Lemma 3.2. For any $0<R \leq 1$ and $\phi \in \mathcal{T}\left(T\left(\mathbb{R}^{n}\right)\right)$ one has

$$
\phi(t)=\int_{|\omega|=1} d \omega \int_{\mathbb{R}^{n}} K(x-i R \omega+i \omega-t) \phi(x-i R \omega) d x .
$$

Proof. Consider the Fourier transform $\hat{\phi}$ of $\phi$ and note that it can be represented as

$$
\hat{\phi}(\xi)=\int_{\mathbb{R}^{n}} \phi(x) e^{i\langle x, \xi\rangle} d x=\int_{\mathbb{R}^{n}} \phi(x-i R \omega) e^{i\langle x-i R \omega, \xi\rangle} d x .
$$

Thus we get

$$
\begin{gathered}
\int_{|\omega|=1} d \omega \int_{\mathbb{R}^{n}} K(x-i R \omega+i \omega-t) \phi(x-i R \omega) d x \\
=(2 \pi)^{-n} \int_{|\omega|=1} d \omega \int_{\mathbb{R}^{n}} d x \phi(x-i R \omega) \int d \xi e^{i\langle x-i R \omega+i \omega-t, \xi\rangle} / I(\xi) \\
=(2 \pi)^{-n} \int_{|\omega|=1} d \omega \int d \xi \int_{\mathbb{R}^{n}} d x \phi(x-i R \omega) e^{i\langle x-i R \omega, \xi\rangle} e^{i\langle i \omega-t, \xi\rangle} / I(\xi) \\
=(2 \pi)^{-n} \int_{|\omega|=1} d \omega \int d \xi \hat{\phi}(\xi) e^{i\langle i \omega-t, \xi\rangle} / I(\xi) \\
=(2 \pi)^{-n} \int d \xi \int_{|\omega|=1} d \omega \hat{\phi}(\xi) e^{i\langle i \omega-t, \xi\rangle} / I(\xi) \\
=(2 \pi)^{-n} \int d \xi \hat{\phi}(\xi) e^{-i\langle t, \xi\rangle} I(\xi) / I(\xi)=(2 \pi)^{-n} \int d \xi \hat{\phi}(\xi) e^{-i\langle t, \xi\rangle}=\phi(t) .
\end{gathered}
$$

Corollary 3.3. Let $K_{r}(z)=r^{-n} K(z / r)$. Then $K_{r}(z)$ is a rapidly decreasing holomorphic function in $\left\{z \in \mathbb{C}^{n} ;|\operatorname{Im} z / r|^{2}<1+|\operatorname{Re} z / r|^{2}\right\}$ $=\left\{z \in \mathbb{C}^{n} ;|\operatorname{Im} z|^{2}<r^{2}+|\operatorname{Re} z|^{2}\right\}$, and for $0<R \leq r$, the identity

$$
\phi(t)=\int_{|\omega|=1} d \omega \int_{\mathbb{R}^{n}} K_{r}(x-i R \omega+i \omega-t) \phi(x-i R \omega) d x
$$

holds.

Proof. A straightforward calculation yields

$$
\begin{gathered}
\int_{|\omega|=1} d \omega \int_{\mathbb{R}^{n}} K_{r}(x-i R \omega+i r \omega-t) \phi(x-i R \omega) d x \\
=r^{-n} \int_{|\omega|=1} d \omega \int_{\mathbb{R}^{n}} K_{1}((x-i R \omega+i r \omega-t) / r) \phi(x-i R \omega) d x
\end{gathered}
$$




$$
\begin{gathered}
=\int_{|\omega|=1} d \omega \int_{\mathbb{R}^{n}} K_{1}(y-i R \omega / r+i \omega-t / r) \phi(r(y-i R \omega / r)) d y \\
=\phi(r(t / r))=\phi(t) .
\end{gathered}
$$

Thus we can remove the restriction $R \leq 1$.

Definition 3.4. Let $O$ be an open set in $\mathbb{R}^{n}$. Denote by $\mathcal{O}_{0}(T(O))$ the set of those functions $F(z)$ which are holomorphic in $T(O)$ and which satisfy the following condition:

For any compact set $K \subset O$, there exists a natural number $j>0$ such that

$$
\sup _{z \in T(K)}|F(z)|(1+|z|)^{-j}<\infty
$$

Now we can state the global form of the edge of the wedge theorem for tempered ultra-hyperfunctions.

Theorem 3.5. Let $V_{+}=\left\{y \in \mathbb{R}^{n} ; y_{1}>\sqrt{\sum_{j=2}^{n} y_{j}^{2}}\right\}$ be the forward light-cone in $\mathbb{R}^{n}, e=(1,0, \ldots, 0) \in \mathbb{R}^{n}$ and $\Gamma=\ell e+V_{+}$. Let $F_{1}(z) \in$ $\mathcal{O}_{0}(T(\Gamma)), F_{2}(z) \in \mathcal{O}_{0}(T(-\Gamma))$ and $C_{j}^{r}=\left\{z_{j} \in \mathbb{C} ; z_{j}=x+i r,-\infty<\right.$ $x<\infty\}$. Define

$$
\begin{aligned}
& u_{1}(\phi)=\int_{\prod_{j} C_{j}^{\eta_{j}}} F_{1}(z) \phi(z) d z, \\
& u_{2}(\phi)=\int_{\prod_{j} C_{j}^{-\eta_{j}}} F_{2}(z) \phi(z) d z
\end{aligned}
$$

for $\phi \in \mathcal{T}\left(T\left(\mathbb{R}^{n}\right)\right)$ and $\eta=\left(\eta_{1}, \ldots, \eta_{n}\right) \in \Gamma$. If $u_{1}=u_{2}$, then $F_{1}(z)$ and $F_{2}(z)$ can be continued analytically to each other and define an entire function.

Proof. Note first that $u_{1}(\phi)$ (resp. $u_{2}(\phi)$ ) does not depend on the path $C_{j}^{\eta_{j}}$ (resp. $C_{j}^{-\eta_{j}}$ ). This can be proved by applying Cauchy's integral theorem $n$ times. Let $r>\ell$. Observe that the function $U_{1}$ defined by

$$
U_{1}(z)=u_{1} * K_{r}(z)=\int_{\mathbb{R}^{n}} F_{1}(\xi+i \eta) K_{r}(z-\xi-i \eta) d \xi
$$

for $\eta \in \Gamma$, is analytic in

$$
\begin{aligned}
V_{1}= & \cup_{\eta \in \Gamma}\left\{z \in \mathbb{C}^{n} ;|\operatorname{Im}(z-i \eta)|<r\right\}=\left\{z \in \mathbb{C}^{n} ; \operatorname{dist}(\operatorname{Im} z, \Gamma)<r\right\} \\
& \supset\left\{z \in \mathbb{C}^{n} ; \operatorname{dist}(\operatorname{Im} z, \ell e)<r\right\} \supset\left\{z \in \mathbb{C}^{n} ;|\operatorname{Im} z|<r-\ell\right\}
\end{aligned}
$$

and similarly $U_{2}(z)=u_{2} * K_{r}(z)$ is analytic in

$$
\begin{aligned}
V_{2}= & \cup_{\eta \in-\Gamma}\left\{z \in \mathbb{C}^{n} ;|\operatorname{Im}(z-i \eta)|<r\right\}=\left\{z \in \mathbb{C}^{n} ; \operatorname{dist}(\operatorname{Im} z,-\Gamma)<r\right\} \\
& \supset\left\{z \in \mathbb{C}^{n} ; \operatorname{dist}(\operatorname{Im} z,-\ell e)<r\right\} \supset\left\{z \in \mathbb{C}^{n} ;|\operatorname{Im} z|<r-\ell\right\} .
\end{aligned}
$$

Corollary 3.3 implies

$$
\int_{|\omega|=1} d \omega \int d x U_{j}(x+i(r-R) \omega) \phi(x-i R \omega)
$$




$$
\begin{gathered}
=\int_{|\omega|=1} d \omega \int d x u_{j} * K_{r}(x+i(r-R) \omega) \phi(x-i R \omega) \\
=\int_{|\omega|=1} d \omega \int d x \int d \xi F_{j}(\xi+i \eta) K_{r}(x+i(r-R) \omega-\xi-i \eta) \phi(x-i R \omega) \\
=\int d \xi \int_{|\omega|=1} d \omega \int d x F_{j}(\xi+i \eta) K_{r}(x+i(r-R) \omega-\xi-i \eta) \phi(x-i R \omega) \\
=\int d \xi F_{j}(\xi+i \eta) \phi(\xi+i \eta)=u_{j}(\phi) .
\end{gathered}
$$

The relation $u_{1}(\phi)=u_{2}(\phi)$ implies $U_{1}(z)=U_{2}(z)$ in $V_{1} \cap V_{2} \supset\{z \in$ $\left.\mathbb{C}^{n} ;|\operatorname{Im} z|<r-\ell\right\}$. Then $U_{1}(z)$ and $U_{2}(z)$ are continued to an analytic function $U(z)$ in $V_{1} \cup V_{2}$. Moreover, $U(z)$ is analytically continued to the convex envelope of $V_{1} \cup V_{2}$ by Bochner's theorem on tublar domains (see [25]). Since the convex envelope of $V_{1} \cup V_{2}$ is entire space $\mathbb{C}^{n}, U(z)$ is analytically continued to an entire function. The function $H$,

$$
H(z)=\int_{|\omega|=1} d \omega U(z+i r \omega),
$$

is an entire function which satisfies

$$
\begin{gathered}
\int_{\prod_{j} C^{\eta_{j}}} H(z) \phi(z) d z=\int_{\prod_{j} C^{\eta_{j}}} d z \int_{|\omega|=1} d \omega U(z+i r \omega) \phi(z) \\
=\int_{|\omega|=1} d \omega \int_{\mathbb{R}^{n}} d x U(x+i \eta+i r \omega) \phi(x+i \eta) \\
=\int_{|\omega|=1} d \omega \int_{\mathbb{R}^{n}} d x U(x+i(r-[\ell+\sigma]) \omega) \phi(x-i[\ell+\sigma] \omega) \\
\left.=\int_{|\omega|=1} d \omega \int_{\mathbb{R}^{n}} d x U_{1}(x+i(r-[\ell+\sigma]) \omega) \phi(x-i[\ell+\sigma]) \omega\right) \\
=u_{1}(\phi)=\int_{\prod_{j} C^{\eta_{j}}} F_{1}(z) \phi(z) d z
\end{gathered}
$$

for $0<\sigma \leq r-\ell$, where we used the fact that $U(z)=U_{1}(z)$ in $\left\{z \in \mathbb{C}^{n} ;|\operatorname{Im} z|<r-\ell\right\}$ and Relation (3.1). Thus we have

$$
\int_{\prod_{j} C^{\eta_{j}}} H(z) \phi(z) d z=\int_{\prod_{j} C^{\eta_{j}}} F_{1}(z) \phi(z) d z
$$

for $\phi \in \mathcal{T}\left(T\left(\mathbb{R}^{n}\right)\right)$. This shows that $H(z)=F_{1}(z)$ in $T(\Gamma)$, and in the same way we get $H(z)=F_{2}(z)$ in $T(-\Gamma)$. Thus $F_{1}(z)$ and $F_{2}(z)$ are analytically continued to an entire function $H(z)$. This completes the proof. 


\section{LOCAL EDGE OF THE WEDGE THEOREM}

We begin by proving a regularization result for tempered ultrahyperfunctions using the kernel $K_{r}$.

Proposition 4.1. Let $L$ be an open set in $\left\{w \in \mathbb{C}^{n} ;|\operatorname{Im} w|<r\right\}$ and $u \in \mathcal{T}(L)^{\prime}$. Then $U(z)=K_{r} * u(z)$ is holomorphic in

$$
Z=\left\{z \in \mathbb{C}^{n} ;|\operatorname{Im}(z-w)|^{2}<r^{2}+|\operatorname{Re}(z-w)|^{2}, \forall w \in L\right\} .
$$

Furthermore, introduce the function

$$
g_{r}(x)=\inf \left\{\sqrt{r^{2}+|x-\operatorname{Re} w|^{2}}-|\operatorname{Im} w| ; w \in L\right\} .
$$

Then the following inclusion is valid.

$$
Z \supset\left\{z=x+i y \in \mathbb{C}^{n} ;|y|<g_{r}(x), x \in \mathbb{R}^{n}\right\} .
$$

Proof. Since $K_{r}(z)$ is a rapidly decreasing holomorphic function in $\{z \in$ $\left.\mathbb{C}^{n} ;|\operatorname{Im} z|^{2}<r^{2}+|\operatorname{Re} z|^{2}\right\}$, if $z \in Z, K_{r}(z-w)$ is a rapidly decreasing holomorphic function of $w$ in a neighborhood of $L$. The inclusion (4.2) can be shown as follows:

$$
\begin{gathered}
Z=\left\{z \in \mathbb{C}^{n} ;|\operatorname{Im}(z-w)|<\sqrt{r^{2}+|\operatorname{Re}(z-w)|^{2}}, \forall w \in L\right\} \\
\supset\left\{z \in \mathbb{C}^{n} ;|\operatorname{Im} z|+|\operatorname{Im} w|<\sqrt{r^{2}+|\operatorname{Re}(z-w)|^{2}}, \forall w \in L\right\} \\
\supset\left\{z=x+i y \in \mathbb{C}^{n} ;|y|<g_{r}(x), x \in \mathbb{R}^{n}\right\} .
\end{gathered}
$$

Theorem 4.2. Let $F_{i}(z)(i=1,2)$ be holomorphic functions and $u_{i}$ be the tempered ultra-hyperfunctions defined by $F_{i}(z)$ as in Theorem 3.5 . Let $L$ be an open set in $\left\{w \in \mathbb{C}^{n} ;|\operatorname{Im} w|<\ell\right\}$ such that the set $O=\{x \in$ $\left.\mathbb{R}^{n} ; g_{r}(x)>r\right\}$ contains an open set $Q$ such that $\operatorname{dist}(\partial O, Q)>2 \ell$, for $r>\ell /(\sqrt{2}-1)$ and $g_{r}(x)$ of $\left.\sqrt{4.1}\right)$. Assume that $u_{1}-u_{2} \in \mathcal{T}(L)^{\prime}$. Then $F_{i}(z)$ are analytically continued to $O$ and coincide there.

Proof. We use the same notation as in Theorem 3.5. Then $U_{1}(z)=$ $u_{1} * K_{r}(z)$ is analytic in

$$
V_{1}=\left\{z \in \mathbb{C}^{n} ; \operatorname{dist}(\operatorname{Im} z, \Gamma)<r\right\}
$$

and $U_{2}(z)=u_{2} * K_{r}(z)$ is analytic in

$$
V_{2}=\left\{z \in \mathbb{C}^{n} ; \operatorname{dist}(\operatorname{Im} z,-\Gamma)<r\right\} .
$$

Since $u_{1}-u_{2} \in \mathcal{T}(L)^{\prime}$, it follows from Proposition 4.1 that $U_{12}(z)=$ $\left(u_{1}-u_{2}\right) * K_{r}(z)$ is analytic in

$$
\supset\left\{z=x+i y \in \mathbb{C}^{n} ;|y|<g_{r}(x), x \in \mathbb{R}^{n}\right\} .
$$

Since $u_{1} * K_{r}(z)=u_{2} * K_{r}(z)+\left(u_{1}-u_{2}\right) * K_{r}(z), U_{1}(z)$ is analytic in

$$
\begin{gathered}
V_{1} \cup\left(V_{2} \cap\left\{z=x+i y \in \mathbb{C}^{n} ;|y|<g_{r}(x), x \in \mathbb{R}^{n}\right\}\right) \\
\supset\left\{x \in \mathbb{R}^{n} ; g_{r}(x)>r+\delta\right\} \\
\times i\left(\left\{y \in \mathbb{R}^{n} ; \operatorname{dist}(y, \Gamma)<r\right\} \cup\left(\left\{y \in \mathbb{R}^{n} ; \operatorname{dist}(y,-\Gamma)<r\right\} \cap B_{r+\delta}\right)\right)
\end{gathered}
$$




$$
=\left\{x \in \mathbb{R}^{n} ; g_{r}(x)>r+\delta\right\} \times i\left(\left\{y \in \mathbb{R}^{n} ; \operatorname{dist}(y, \Gamma)<r\right\} \cup B_{r+\delta}\right)
$$

for any $r+\delta<\sqrt{2} r+\ell$, where $B_{r}=\left\{y \in \mathbb{R}^{n} ;|y|<r\right\}$ (see Figure $2)$. Note that if $r>\ell /(\sqrt{2}-1)$ then there exists $\delta>0$ such that $r+\delta<\sqrt{2} r+\ell$.

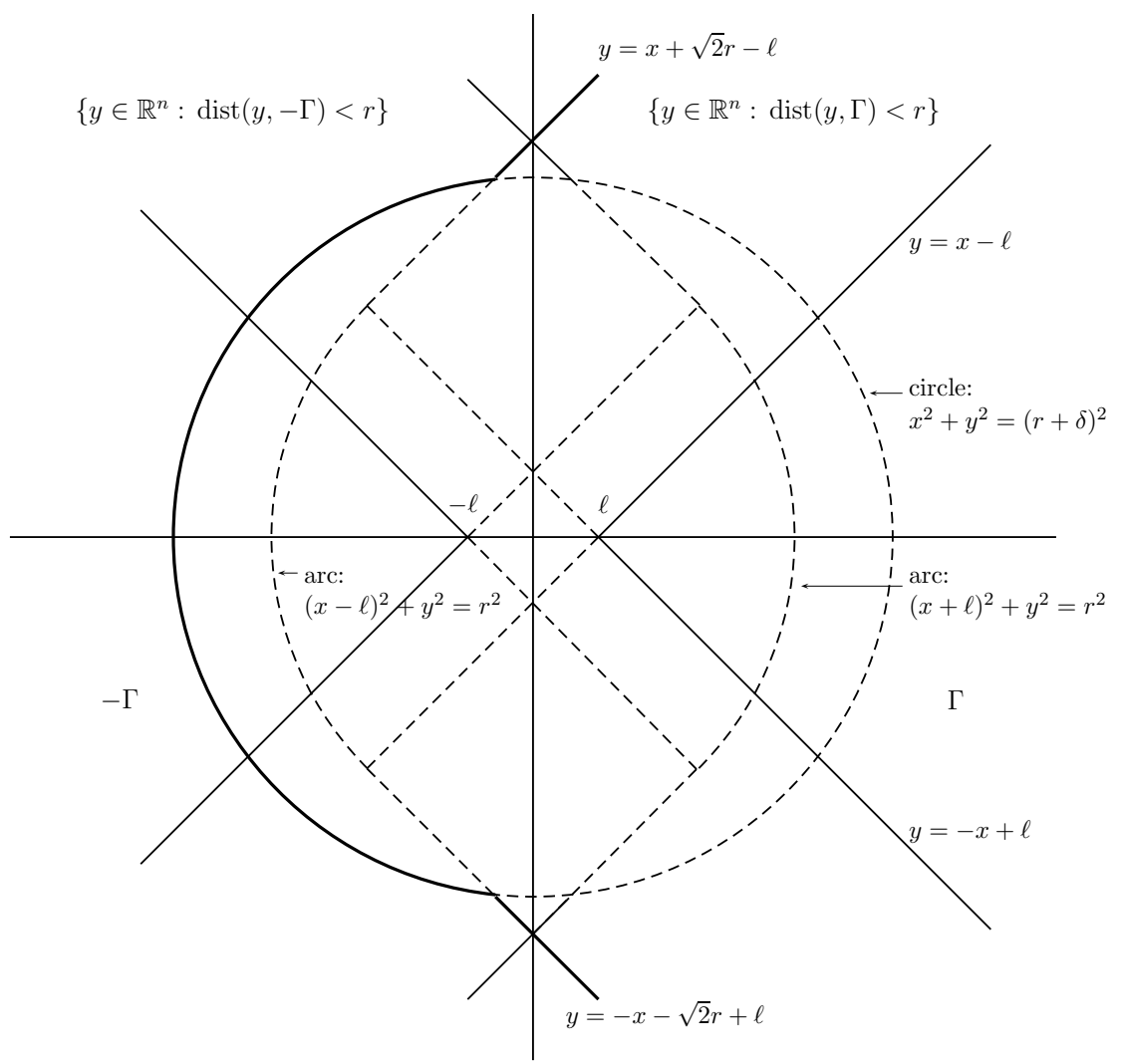

Figure 2. $\left\{y \in \mathbb{R}^{n} ; \operatorname{dist}(y, \Gamma)<r\right\} \cup(\{y \in$ $\left.\left.\mathbb{R}^{n} ; \operatorname{dist}(y,-\Gamma)<r\right\} \cap B_{r+\delta}\right)=\left\{y \in \mathbb{R}^{n} ; \operatorname{dist}(y, \Gamma)<\right.$ $r\} \cup B_{r+\delta}$

Denote

$$
W_{r, \delta}=\left\{y \in \mathbb{R}^{n} ; \operatorname{dist}(y, \Gamma)<r\right\} \cup B_{r+\delta}
$$

If

$$
r+\delta>\sqrt{(r / \sqrt{2})^{2}+(r / \sqrt{2}-\ell)^{2}}
$$

then $\cap_{|\omega|=1}\left(W_{r, \delta}+r \omega\right)$ strictly contains the set $\Gamma$, and if $\delta>0$ then $\cap_{|\omega|=1}\left(W_{r, \delta}+r \omega\right)$ contains a connected set $\tilde{\Gamma}_{\delta}$ containing $\Gamma$ and an open ball $B_{\delta}$ (see Figure 3 ). 


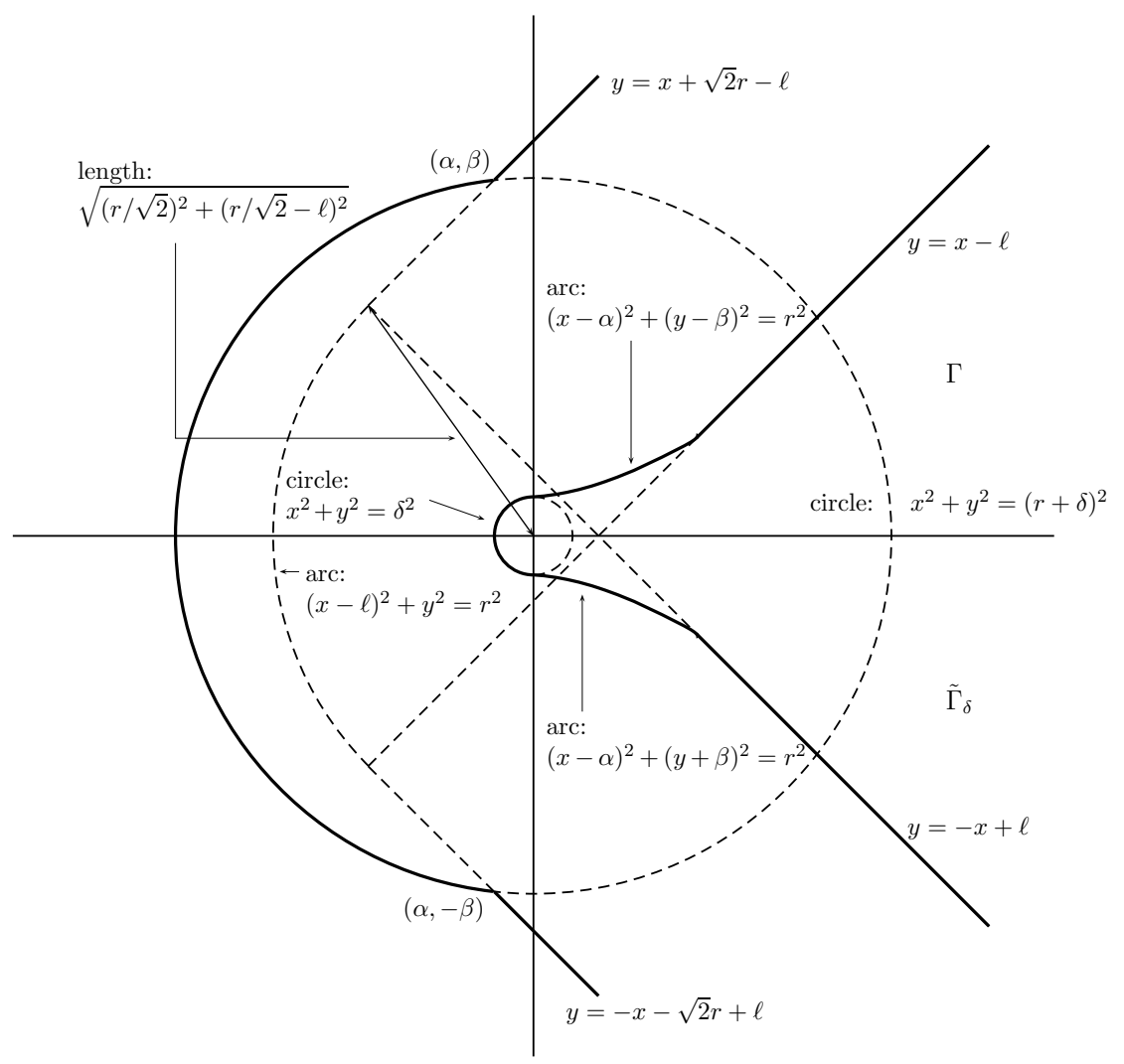

Figure 3. The connected set $\tilde{\Gamma}_{\delta}$ containing $\Gamma$ and $B_{\delta}$

As before, consider

$$
H_{1}(z)=\int_{|\omega|=1} d \omega U_{1}(z+i r \omega)
$$

$H_{1}(z)$ is holomorphic in the set

$$
\cap_{|\omega|=1}\left[\left\{V_{1} \cup\left(V_{2} \cap\left\{z=x+i y \in \mathbb{C}^{n} ;|y|<g_{r}(x), x \in \mathbb{R}^{n}\right\}\right)\right\}+i r \omega\right]
$$

which contains the sets $T(\Gamma)$ and

$\left\{x \in \mathbb{R}^{n} ; g_{r}(x)>r+\delta\right\} \times i \cap_{|\omega|=1}\left(W_{r+\delta}+r \omega\right) \supset\left\{x \in \mathbb{R}^{n} ; g_{r}(x)>r+\delta\right\} \times i \tilde{\Gamma}_{\delta}$.

This implies

$$
\begin{gathered}
\int_{\prod_{j} C^{\eta_{j}}} H_{1}(z) \phi(z) d z=\int_{\prod_{j} C^{\eta_{j}}} d z \int_{|\omega|=1} d \omega U_{1}(z+i r \omega) \phi(z) \\
=\int_{|\omega|=1} d \omega \int_{\mathbb{R}^{n}} d y U_{1}(y+i \eta+i r \omega) \phi(y+i \eta) \\
=\int_{|\omega|=1} d \omega \int_{\mathbb{R}^{n}} d y U_{1}(y+i(r-[\ell+\sigma]) \omega) \phi(y-i[\ell+\sigma] \omega) \\
=u_{1}(\phi)=\int_{\prod_{j} C^{\eta_{j}}} F_{1}(z) \phi(z) d z
\end{gathered}
$$


This shows

$$
\int_{\prod_{j} C^{\eta_{j}}} H_{1}(z) \phi(z) d z=\int_{\prod_{j} C^{\eta_{j}}} F_{1}(z) \phi(z) d z
$$

for $\phi \in \mathcal{T}\left(T\left(\mathbb{R}^{n}\right)\right)$ and therefore $H_{1}(z)=F_{1}(z)$ in $T(\Gamma)$ and $F_{1}(z)$ is analytically continued to

$$
\left\{x \in \mathbb{R}^{n} ; g_{r}(x)>r+\delta\right\}+i \tilde{\Gamma}_{\delta} .
$$

In the same way we get $H_{2}(z)=F_{2}(z)$ in $T(-\Gamma)$ and $F_{2}(z)$ is analytically continued to

$$
\left\{x \in \mathbb{R}^{n} ; g_{r}(x)>r+\delta\right\}-i \tilde{\Gamma}_{\delta} .
$$

In order to show $H_{1}(z)=H_{2}(z)$, we choose a surface $S_{1}$ contained in the domain of the holomorphy of $H_{1}(z)$ such that

$$
S_{1}=\left\{z=x+i y \in \mathbb{C}^{n} ; y_{1}=f_{1}(x), y_{j}=0,2 \leq j \leq n, x \in \mathbb{R}^{n}\right\},
$$

where $0 \leq f_{1}(x) \leq \ell+\delta$ is a continuous function satisfying $f_{1}(x)=0$ for $x \in O=\left\{x \in \mathbb{R}^{n} ; g_{r}(x)>r\right\}$ and $f_{1}(x)=\ell+\delta$ for

$$
x \in\left\{x \in \mathbb{R}^{n} ; g_{r}(x)<\sqrt{(r / \sqrt{2})^{2}+(r / \sqrt{2}-\ell)^{2}}\right\} .
$$

For $\xi \in Q \subset O$ define $E_{\xi}^{t}(z)=(4 \pi t)^{-n / 2} e^{-(\xi-z)^{2} / 4 t}$. Note that the open ball with center $\xi$ and radius $2 \ell$ is contained in $O, B_{2 \ell}(\xi) \subset O \subset S_{1}$. Then, for $\xi \in Q$,

$$
\begin{gathered}
u_{1}\left(E_{\xi}\right)=\int_{S_{1}} H_{1}(z) E_{\xi}^{t}(z) d z \\
=\int_{B_{2 \ell}(\xi)} H_{1}(z) E(\xi-z) d z+\int_{S_{1} \backslash B_{2 \ell}(\xi)} H_{1}(z) E_{\xi}^{t}(z) d z
\end{gathered}
$$

and

$$
\int_{B_{2 \ell}(\xi)} H_{1}(z) E_{\xi}^{t}(z) d z=\int_{B_{2 \ell}(\xi)} H_{1}(z)(4 \pi t)^{-n / 2} e^{-(\xi-x)^{2} / 4 t} d x \rightarrow H_{1}(\xi)
$$

as $t \rightarrow 0+$. Since

$$
\int_{S_{1} \backslash B_{2 \ell}(\xi)} H_{1}(z) E_{\xi}^{t}(z) d z=\int_{S_{1} \backslash B_{2 \ell}(\xi)} H_{1}(z)(4 \pi t)^{-n / 2} e^{-(\xi-z)^{2} / 4 t} d z
$$

and for $z \in S_{1} \backslash B_{2 \ell}(\xi)$,

$$
\left|e^{-(\xi-z)^{2} / 4 t}\right|=e^{-(\xi-x)^{2} / 4 t} e^{y_{1}^{2} / 4 t}\left|e^{-2 i\left(\xi_{1}-x_{1}\right) y_{1} / 4 t}\right| \leq e^{-(\xi-x)^{2} / 4 t} e^{(\ell+\delta)^{2} / 4 t},
$$

we get

$$
\int_{S_{1} \backslash B_{2 \ell}(\xi)} H_{1}(z) E_{\xi}^{t}(z) d z \rightarrow 0
$$

as $t \rightarrow 0+$, where we used the relation $(\xi-x)^{2} \geq 4 \ell^{2}$ and consequently, $(\xi-x)^{2} / 2 \geq(\ell+\delta)^{2}$ for $0<\delta<\ell / 2$. It follows

$$
\lim _{t \rightarrow 0} u_{1}\left(E_{\xi}^{t}\right)=H_{1}(\xi) \text {. }
$$


In the same way we get

$$
\lim _{t \rightarrow 0} u_{2}\left(E_{\xi}^{t}\right)=H_{2}(\xi)
$$

Let $x \in O=\left\{x \in \mathbb{R}^{n} ; g_{r}(x)>r\right\}$. Then

$$
\sqrt{r^{2}+|x-\operatorname{Re} w|^{2}}-|\operatorname{Im} w|>r
$$

for any $w \in L$, that is, $|x-\operatorname{Re} w|>0$. Let $\xi \in Q$. Then $|\xi-\operatorname{Re} w|>2 \ell$ for any $w \in L$ and

$$
\begin{gathered}
\sup _{w \in L}(1+|w|)^{j}(4 \pi t)^{-n / 2} \exp \left[-(\xi-\operatorname{Re} w)^{2}+(\operatorname{Im} w)^{2}\right] / 4 t \\
\leq \sup _{w \in L}(1+|w|)^{j}(4 \pi t)^{-n / 2} \exp \left[-(\xi-\operatorname{Re} w)^{2} / 2-\ell^{2}\right] / 4 t \rightarrow 0 \\
\text { as } t \rightarrow 0+. u_{1}-u_{2} \in \mathcal{T}(L)^{\prime} \text { implies } \\
0=\lim _{t \rightarrow 0}\left(u_{1}-u_{2}\right)\left(E_{\xi}^{t}\right)=\lim _{t \rightarrow 0} u_{1}\left(E_{\xi}^{t}\right)-\lim _{t \rightarrow 0} u_{2}\left(E_{\xi}^{t}\right)=H_{1}(\xi)-H_{2}(\xi) .
\end{gathered}
$$

Thus $H_{1}(z)$ and $H_{2}(z)$ are analytically continued to each other. This completes the proof.

The following corollary is used in [21].

Corollary 4.3. Let $V_{+}=\left\{y \in \mathbb{R}^{4} ; y_{0}>\sqrt{\sum_{j=1}^{3} y_{j}^{2}}\right\}$ be the forward light-cone in $\mathbb{R}^{4}, e=(1,0,0,0) \in \mathbb{R}^{4}$ and $\Gamma=\ell e+V_{+}$. Let $F_{1}(z) \in$ $\mathcal{O}_{0}(T(\Gamma)), F_{2}(z) \in \mathcal{O}_{0}(T(-\Gamma))$ and $C_{j}^{r}=\left\{z_{j} \in \mathbb{C} ; z_{j}=x+i r,-\infty<\right.$ $x<\infty\}$. Define

$$
\begin{aligned}
u_{1}(\phi) & =\int_{\prod_{j} C_{j}^{\eta_{j}}} F_{1}(z) \phi(z) d z, \\
u_{2}(\phi) & =\int_{\prod_{j} C_{j}^{-\eta_{j}}} F_{2}(z) \phi(z) d z
\end{aligned}
$$

for $\phi \in \mathcal{T}\left(T\left(\mathbb{R}^{4}\right)\right)$ and $\eta=\left(\eta_{0}, \ldots, \eta_{3}\right) \in \Gamma$. Let

$$
L=\left\{w \in \mathbb{C}^{4} ; \exists x \in V|\operatorname{Re} w-x|+|\operatorname{Im} w|_{1}<\ell\right\},
$$

where $V$ is the light-cone and $|y|_{1}=\left|y^{0}\right|+|\boldsymbol{y}|$. Assume that $u_{1}-u_{2} \in$ $\mathcal{T}(L)^{\prime}$. Then $F_{i}(z)(i=1,2)$ are analytically continued to the set

$$
\left\{x \in \mathbb{R}^{4} ; \operatorname{dist}(x, V)>(\sqrt{2}+1) \ell\right\}
$$

and coincide there.

Proof. The corollary follows from Theorem 4.2 and the following lemma.

Lemma 4.4. Let $L$ be the set defined in the above corollary and $g_{r}(x)$ the function defined by 4 4.1) with $r>\ell /(\sqrt{2}-1)$. Then the set $O=$ $\left\{x \in \mathbb{R}^{4} ; g_{r}(x)>r\right\}$ contains a set

$$
\left\{x \in \mathbb{R}^{4} ; \operatorname{dist}(x, V)>(\sqrt{2}+1) \ell\right\} .
$$


The open set

$$
Q=\left\{x \in \mathbb{R}^{4} ; \operatorname{dist}(x, V)>(\sqrt{2}+3) \ell\right\}
$$

is contained in $O$ and dist $(\partial O, Q)>2 \ell$.

Proof. Observe that

$$
\begin{aligned}
g_{r}(x) & \leq \inf \left\{\sqrt{r^{2}+|x-\operatorname{Re} w|^{2}}-|\operatorname{Im} w| ;(w \in L) \wedge(\operatorname{Re} w \in V)\right\} \\
& =\inf \left\{\sqrt{r^{2}+|x-u|^{2}}-\ell ; u \in V\right\}=\sqrt{r^{2}+y^{2}}-\ell,
\end{aligned}
$$

where $y=\operatorname{dist}(x, V)$. Then $g_{r}(x)>r$ implies $\sqrt{r^{2}+y^{2}}>r+\ell$ and $y^{2}>\ell(2 r+\ell)$. Since we can choose $r>\ell /(\sqrt{2}-1)$ arbitrarily close to $\ell /(\sqrt{2}-1)$, we have $y^{2}>(\sqrt{2}+1)^{2} \ell^{2}$. This completes the proof.

Remark 4.5. According to Proposition 4.7 of [3] the $n$-point function in the difference variables $W_{n-1}\left(\zeta_{1}, \ldots, \zeta_{n-1}\right)$ is analytic in

$$
\left\{\zeta \in \mathbb{C}^{4(n-1)} ; \operatorname{Im} \zeta_{j} \in V_{+}+\ell^{\prime} e, \operatorname{Im} \zeta_{k} \in V_{+}+R e, \ell<\ell^{\prime}, k \neq j\right\}
$$

for sufficiently large $R>0$. Now for

$$
g_{n-2}^{j}\left(\zeta_{1}, \ldots, \zeta_{j-1}, \hat{\zeta}_{j}, \zeta_{j+1}, \ldots, \zeta_{n-1}\right) \in \mathcal{T}\left(T\left(\mathbb{R}^{4(n-2)}\right)\right),
$$

define

$$
\begin{gathered}
F_{1}\left(\zeta_{j}\right)=W_{n-1}\left(\zeta_{j}, g_{n-2}^{j}\right) \\
=\left\langle W_{n-1}\left(\zeta_{1}, \ldots, \zeta_{n-1}\right), g_{n-2}^{j}\left(\zeta_{1}, \ldots, \zeta_{j-1}, \hat{\zeta}_{j}, \zeta_{j+1}, \ldots, \zeta_{n-1}\right)\right\rangle, \\
F_{2}\left(\zeta_{j}\right)=W_{n-1}^{j}\left(\zeta_{j}, g_{n-2}^{j}\right) \\
=\left\langle W_{n-1}^{j}\left(\zeta_{1}, \ldots, \zeta_{n-1}\right), g_{n-2}^{j}\left(\zeta_{1}, \ldots, \zeta_{j-1}, \hat{\zeta}_{j}, \zeta_{j+1}, \ldots, \zeta_{n-1}\right)\right\rangle,
\end{gathered}
$$

where

$$
W_{n-1}^{j}\left(\zeta_{1}, \ldots, \zeta_{n-1}\right)=W_{n-1}\left(\zeta_{1}, \ldots, \zeta_{j-1}+\zeta_{j},-\zeta_{j}, \zeta_{j}+\zeta_{j+1}, \ldots, \zeta_{n-1}\right) .
$$

Then $F_{1}$ and $F_{2}$ satisfy the assumptions of Corollary 4.3 and $F_{i}(z)$ $(i=1,2)$ are analytically continued to the set (4.3) and coincide there. But in [21] it is shown that

$$
W_{n-1}\left(\xi_{j}, g_{n-2}^{j}\right)-W_{n-1}^{j}\left(\xi_{j}, g_{n-2}^{j}\right)=0
$$

in the set

$$
\left\{x \in \mathbb{R}^{n} ; \operatorname{dist}(x, \bar{V})>\ell\right\} .
$$

So, we might expect that $F_{i}(z)(i=1,2)$ are analytically continued to each other through the set (4.4). We can show that $u_{1}-u_{2}$ is analytic in the set (4.4) as follows. If $x$ belongs to the set (4.4), then there exists $\delta>0$ such that $|x-\operatorname{Re} w|>|\operatorname{Im} w|+\delta$ for all $w \in L$, and therefore we have $g_{r}(x)>r+\delta$ for sufficiently small $r>0$ (see (4.1)). Then $U_{12}(z)=\left(u_{1}-u_{2}\right) * K_{r}(z)$ is holomorphic in the set (4.2) which contains the set

$$
\left\{z=x+i y \in \mathbb{C}^{n} ;|y|<r+\delta, \operatorname{dist}(x, \bar{V})>\ell\right\},
$$


(see (4.2)) and therefore

$$
H_{12}(z)=\int_{|\omega|=1} d \omega U_{12}(z+i r \omega)
$$

is holomorpic in the set

$$
\left\{z=x+i y \in \mathbb{C}^{n} ;|y|<\delta, \operatorname{dist}(x, \bar{V})>\ell\right\},
$$

which shows that $u_{1}-u_{2}$ is analytic in the set (4.4). But in order to show that $F_{i}(z)(i=1,2)$ are analytically continued to each other by our method, $V_{1} \cup V_{2}$ must contain $B_{r+\delta}$ for some $\delta>0$, and therefore $\sqrt{2} r-\ell>r$ must be hold (see figure 2), i.e., $r>\ell /(\sqrt{2}-1)$. This is the reason why our method can only show that $F_{i}(z)(i=1,2)$ are analytically continued to each other through the set (4.3).

\section{REFERENCES}

1 N. N. Bogoliubov and V. S. Vladimirov. On some mathematical problem of quantized field theory. In Proceedings of the International Congress of Mathematicians, Edinburgh 1958, New York, 1960. Cambridge University Press.

2 E. Brüning and S. Nagamachi. Hyperfunction quantum field theory: Basic structural results. J. Math. Physics, 30:2340-2359, 1989.

3 E. Brüning and S. Nagamachi. Relativistic quantum field theory with a fundamental length. J. Math. Phys., 45:2199-2231, 2004.

4 E. Brüning and S. Nagamachi. Solutions of a linearized model of Heisenberg's fundamental equation II. J. Math. Phys., 49:052304-1 - 052304-22, 2008.

${ }^{5}$ H. Epstein. Generalization of the "edge of the wedge" theorem. J. Math. Phys., 1:524-531, 1960.

6 D. H.T. Franco. The edge of the wedge theorem for tempered ultrahyperfuncions. arXiv:math/0609751, 2006.

7 D. H.T. Franco. The edge of the wedge theorem for tempered ultrahyperfuncions II, a generalized version. arXiv:0708.0252, 2007.

8 M. Hasumi. Note on the $n$-dimensional tempered ultra-distributions. Tohoku Math. J., 13:94-104, 1961.

9 L. Hörmander. The analysis of linear partial differential operators I, volume 256 of Grundlehren der mathematischen Wissenschaften. Springer-Verlag, Berlin Heidelberg New York Tokyo, 1983.

10 A. Kaneko. Introduction to Hyperfunctions. Mathematics and Its Applicatons (Japanese Series). Kluwer Academic Publishers, Dordrecht Boston London, 1988.

11 T. Kawai. On the theory of Fourier hyperfunctions and its applications to partial differential equations with constant coefficients. J. Fac. Sci. Univ. Tokyo, Sect. I.A, 17:467-517, 1970.

12 A. Martineau. Distributions et valeurs au bord des fonctions holomorphes. In Proc. Intern. Summer Course on the Theory of Distributions, pages 195-326, Lisbon, 1964.

13 A. Martineau. Théorème sur le prolongement analytique du type "Edge of the Wedge Theorem". Séminair Bourbaki, 20-ième année, No. 340, 1967/68.

14 A. Martineau. Le "edge of the wedge theorem" en théorie des hyperfonctions de sato. In Proc. Intern. Conf. on Functional Analysis, Tokyo, 1969, pages 95-106, Tokyo, 1970. Univ. Tokyo Press. 
15 M. Morimoto. Sur les ultradistributions cohomologiques. Ann. Inst. Fourier, 19:129-153, 1969.

16 M. Morimoto. La décomposition de singularités d'ultradistributions cohomologiques. Proc. Japan Acad., 48:129-153, 1972.

17 M. Morimoto. Edge of the wedge theorem and hyperfunction. In Hyperfunctions and pseudo-differential equations (Proc. Conf., Katata, 1971, pages 41-81, Berlin, 1973. Lecture Notes in Math., Vol. 287, Springer.

18 M. Morimoto. Convolutors for ultrahyperfunctions. In International Symposium on Mathematical Problems in Theoretical Physics, volume 39 of Lecture Notes in Phys., pages 49-54, Berlin, 1975. Springer.

19 M. Morimoto. An introduction to Sato's hyperfunctions. American Mathematical Society, 1993.

20 S. Nagamachi and E. Brüning. Solutions of a linearized model of Heisenberg's fundamental equation I. arXiv:0804.1663 [math-ph], 2008.

21 S. Nagamachi and E. Brüning. Frame Independence of the Fundamental Length in Relativistic Quantum Field Theory. Preprint, 2009.

22 S. Nagamachi and T. Nishimura. Edge of the wedge theorem for Fourier hyperfunctions. Funkcialaj Ekvacioj, 36:499-517, 1993.

23 T. Nishimura and S. Ngamachi. Support and kernel theorem for Fourier hyperfunctions. Osaka J. Math., 38:667-680, 2001.

24 M. Suwa. Distributions of Exponential Growth with Support in a Proper Cone. Publ. RIMS, Kyoto Univ., 40:565 - 603, 2004.

25 V. S. Vladimirov. Methods of the Theory of Functions of Many Complex Variables. The M.I.T Press, Cambridge, Massachussetts, London, 1964.

(E. Brüning) School of Mathematical Sciences, University of KwaZulu-

Natal, Private Bag X54001, Durban 4000, South Africa

E-mail address: bruninge@ukzn.ac.za

(S. Nagamachi) Emeritus Professor, FACUlty of Engineering, The UniVERsity OF TOKUshima, TOKUSHIMA 770-8506, JAPAN

E-mail address: shigeaki-matematiko@memoad.jp 